\title{
Disorder-enhanced delocalization and local-moment quenching in a disordered antiferromagnet
}

\author{
Avinash Singh, ${ }^{*}$ Martin Ulmke, and Dieter Vollhardt \\ Theoretische Physik III, Elektronische Korrelationen und Magnetismus, Universität Augsburg, D-86135 Augsburg, Germany
}

(Received 6 March 1998)

\begin{abstract}
The interplay of disorder and spin-fluctuation effects in a disordered antiferromagnet is studied. In the weak-disorder regime $(W \leqslant U)$, while the energy gap decreases rapidly with disorder, the sublattice magnetization, including quantum corrections, is found to remain essentially unchanged in the strong correlation limit. Magnon energies and Néel temperature are enhanced by disorder in this limit. A single paradigm of disorderenhanced delocalization qualitatively accounts for all these weak disorder effects. Vertex corrections and magnon damping, which appear only at $\operatorname{order}(W / U)^{4}$, are also studied. With increasing disorder a crossover is found at $W \sim U$, characterized by a rapid decrease in sublattice magnetization due to quenching of local moments, and formation of spin vacancies. The latter suggests a spin-dilution behavior that is indeed observed in softened magnon modes, lowering of Néel temperature, and enhanced transverse spin fluctuations. [S0163-1829(98)00137-4]
\end{abstract}

\section{INTRODUCTION}

The manifestation of quantum antiferromagnetism in parent cuprates, ${ }^{1}$ discovered soon after the birth of high- $T_{\mathrm{c}}$ superconductivity in the doped materials, ${ }^{2}$ has led to intensive efforts to understand the nature of this phase both within the Hubbard model, as well as its strong-coupling counterpart, the quantum Heisenberg model. ${ }^{3}$ Features such as the substantially reduced sublattice magnetization (relative to the classical value) as deduced from neutron-scattering experiments, ${ }^{1}$ the substantially enhanced linewidth observed in the two-magnon Raman scattering experiments, ${ }^{4,5}$ as well as a detailed fitting of the temperature dependence of the spin-correlation length ${ }^{6,7}$ have confirmed the importance of quantum spin fluctuations in these low-dimensional, lowspin systems. Antiferromagnetic (AF) spin correlations are also present in other strongly correlated systems, notably the transition-metal oxides such as $\mathrm{NiO}, \mathrm{V}_{2} \mathrm{O}_{3}, \mathrm{LaVO}_{3}$, $\mathrm{NiS}_{2-x} \mathrm{Se}_{x}$, and heavy-fermion compounds such as $\mathrm{YbP}$, $\mathrm{U}_{2} \mathrm{Zn}_{17}, \mathrm{UCd}_{11}, \mathrm{UCu}_{5}$ that exhibit AF ordering of $d$ and $f$ electrons, respectively, in their ground states. ${ }^{8-11}$

Many of the correlated electron systems are intrinsically disordered, and the metal-insulator transition observed in several amorphous materials, such as doped semiconductors, amorphous $\mathrm{Ge}_{1-x} \mathrm{Au}_{x}$ and $\mathrm{B}_{1-x} \mathrm{Cu}_{x}$ alloys, and granular alumina, have the character of both the Mott transition and the Anderson localization transition. ${ }^{12}$ The role of strong disorder effects has also been emphasized in the recently studied transition-metal oxides such as $\mathrm{LaNi}_{1-x} \mathrm{Co}_{x} \mathrm{O}_{3}$ and $\mathrm{Na}_{x} \mathrm{WO}_{3} .{ }^{13}$ The square-root dip in the electronic density of states near the Fermi energy, characteristic of disorderinduced enhancement of interaction effects, ${ }^{12}$ has been found to change to a linear form and then to a soft quadratic gap on the insulating side. ${ }^{14,13}$ In some cases, such as in $\mathrm{La}_{1-x} \mathrm{Sr}_{x} \mathrm{VO}_{3}, \mathrm{~V}_{2} \mathrm{O}_{3}$, the insulator-metal transition is accompanied with loss of AF order, ${ }^{13,10}$ whereas $\mathrm{NiS}_{2-x} \mathrm{Se}_{x}$ exhibits an AF metallic phase. ${ }^{10}$

It is therefore of interest to study the interplay of quantum spin fluctuation and disorder effects. Of particular interest are questions such as the following: (i) Are transverse spin fluctuations and quantum corrections to the sublattice magnetization enhanced by disorder? (ii) Does a gapless AF state exist, and, if so, at what critical disorder strength is AF longrange order (AFLRO) destroyed? (iii) Is the AF state destabilized by disorder at finite temperature such that the Néel temperature (for dimensions $d>2$ ) is lowered? To answer these questions we will examine the influence of diagonal disorder on various properties of the Hubbard antiferromagnet, such as sublattice magnetization, quantum spin fluctuations, magnon energy and damping, Néel temperature, Hubbard energy gap, and electronic density of states.

Recently spin-fluctuation effects were examined in impurity-doped antiferromagnets, both within the Heisenberg model, ${ }^{15-19}$ as well as the Hubbard model, ${ }^{20-22}$ in order to study magnetic dynamics in cuprate antiferromagnets doped with nonmagnetic and magnetic impurities such as Zn, $\mathrm{Al}, \mathrm{Ga}$, and $\mathrm{Fe}, \mathrm{Ni}, \mathrm{Co}$, respectively. It was found that a static vacancy, created by the replacement of a fermion with a nonmagnetic impurity, for instance, leads to strong magnon scattering. It is therefore also of interest to contrast scattering of magnons caused by disorder with that caused by static vacancies. The third, related, case is that of magnon scattering off mobile vacancies, as in hole-doped cuprates, which is of course much more efficient at destroying AFLRO; spincorrelation lengths of order of $1 / \sqrt{x}$ for hole concentration $x$ have been reported from neutron-scattering studies. ${ }^{23}$

This study therefore complements earlier works on the disordered Hubbard model where other aspects have been studied, such as the metal-insulator transition, ${ }^{24,25}$ localmoment formation, ${ }^{26-28}$ phase diagram, etc. A variety of methods have been used earlier, including the scaling theory, ${ }^{29}$ field-theoretic approaches, ${ }^{30-32}$ renormalization group $(\mathrm{RG}),{ }^{33,34}$ real-space $\quad \mathrm{RG},{ }^{35,36}$ slave-boson formulation, ${ }^{37}$ dynamical mean-field theory, ${ }^{38-40}$ quantum Monte Carlo studies, ${ }^{41}$ and unrestricted Hartree-Fock theory together with random-phase approximation, and Onsagerreaction-field correction to mean-field theory of equivalent spin models. ${ }^{42-44}$ The disordered Hubbard model exhibits extremely rich physics and contains the noninteracting Anderson localization transition, the purely interacting mag- 
netic transition, and of course the nontrivial fixed point describing the metal-insulator transition in the disordered, interacting theory. In addition, various ingredients such as the phenomena of weak localization, disorder-induced enhancement of interaction effects leading to singularities at the Fermi energy, local-moment behavior, etc., are also contained. ${ }^{25}$ Generally, the simultaneous presence of interaction and disorder leads to a new coupling of the quantum degrees of freedom in two-particle quantities that has no counterpart in noninteracting, disordered, or interacting, pure systems.

We consider the following Hubbard Hamiltonian with random on-site energies, and with a filling of one fermion per site, so that an AF ground state is obtained:

$$
H=\sum_{i \sigma} \epsilon_{i} \hat{n}_{i \sigma}-t \sum_{\langle i j\rangle \sigma}\left(\hat{a}_{i \sigma}^{\dagger} \hat{a}_{j \sigma}+\text { H.c. }\right)+U \sum_{i} \hat{n}_{i \uparrow} \hat{n}_{i \downarrow} .
$$

The random on-site energies $\epsilon_{i}$ are chosen from a uniform distribution with $-W / 2<\epsilon_{i}<W / 2$, the distribution width $W$ parametrizing the disorder strength. We consider both the strong correlation limit, with the correlation term $U$ much larger than the free-particle bandwidth $B=2 Z t$, where $Z$ $=2 d$ is the coordination number, as well as the intermediate correlation regime, with $U \sim B$. For concreteness, we consider the square lattice, generalization to three dimensions and to other bipartite lattices being straightforward.

We will use several methods/approximations in this paper. The broken-symmetry state is obtained in the unrestricted Hartree-Fock (UHF) approximation, and transverse spin fluctuations about this state are studied in the randomphase approximation (RPA). Disorder is treated both perturbatively as well as within a numerical diagonalization approach on finite lattices. In the latter approach the eigenfunctions and eigenvalues of the HF Hamiltonian in the fully self-consistent state are used to obtain sublattice magnetization, energy gap, and the magnon spectrum. The $T$-matrix approach, used earlier for impurities ${ }^{20}$ is also employed for comparison. Quantum spin-fluctuation corrections are obtained at the one-loop level. ${ }^{45}$ The Néel temperature, energy gap, and electronic spectrum are also studied within the dynamical mean field theory (DMFT). ${ }^{40}$

The outline of the paper is as follows. Section II deals with the reduction of the Hubbard gap due to formation of disorder-induced states. Disorder renormalizations of the magnon energy, damping, and sublattice magnetization are described in Secs. III and IV, based on results of a perturbative analysis in powers of $W / U$, discussed in the Appendix. A qualitative explanation of the disorder effects is given in Sec. V in terms of the notion of disorder-enhanced delocalization. Section VI describes the crossover to the strong disorder regime $(W>U)$, where the electronic spectrum is gapless, and spin vacancies are formed due to quenching of local moments. Magnon softening, enhancement in transverse spin fluctuation due to spin vacancies, and robustness of the gapless AF state are discussed. Conclusions are given in Sec. VII.

\section{DISORDER-INDUCED STATES IN THE GAP}

In this section we examine the formation of disorderinduced states within the Hubbard gap that reduce the charge

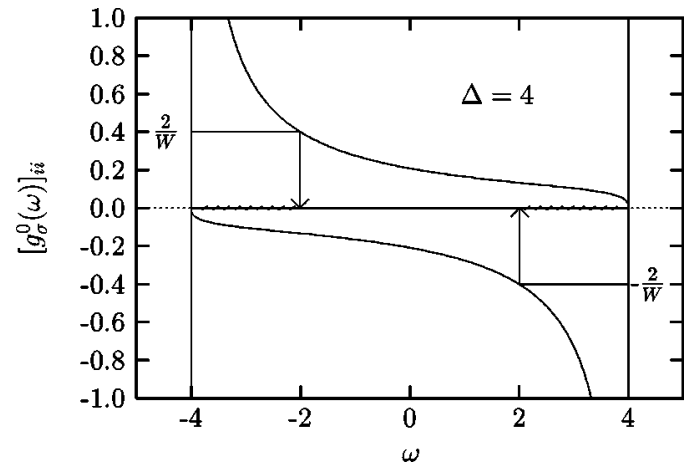

FIG. 1. Local-host Green's function $\left[g_{\sigma}^{0}(\omega)\right]_{i i}$ vs $\omega$ in the pure AF state. Intersections with lines $2 / W$ and $-2 / W$ show the extent to which disorder-induced states are formed within the Hubbard gap. In all figures energies are in units of the hopping parameter $t$.

gap and determine the critical disorder strength at which the gap vanishes. We use the $T$-matrix approach and a numerical UHF approach, described in Secs. II A and II B, respectively. The $T$-matrix approach is exact for a single impurity, and has been used earlier to study the formation of defect states within the gap due to a single nonmagnetic impurity in the Hubbard $\mathrm{AF}^{20}$ In order to use this approach for the disordered $\mathrm{AF}$, with random potentials on every site, we make a local approximation in which we consider a single site, and treat the random potential $\epsilon_{i}$ on this site as an impurity potential. Comparison with results of the numerical UHF analysis, in which disorder is treated exactly, indicates that this approximation actually works quite well, particularly in the strong correlation limit, where states are strongly localized. Spin-fluctuation processes will lead to small changes in the energy gap of order $J$ in the strong-correlation limit.

\section{A. $T$-matrix approach}

Within this approach the location of impurity-induced states is obtained from the pole in the $T$ matrix, $T_{\sigma}(\omega)$ $=\epsilon_{i} /\left(1-\epsilon_{i}\left[g_{\sigma}^{0}(\omega)\right]_{i i}\right)$, where $\left[g_{\sigma}^{0}\right]_{i i}$ is the local-host Green's function. For the pure AF it is given (in the HartreeFock approximation) by $\left[g_{\sigma}^{0}(\omega)\right]_{i i}=(1 / N) \Sigma_{\mathbf{k}}(\omega \mp \sigma \Delta) /\left(\omega^{2}\right.$ $-E_{\mathbf{k}}^{2}$ ), for site $i$ in the $A$ or $B$ sublattice. ${ }^{20}$ Here $2 \Delta=m U$ is the Hubbard energy gap in the pure AF, $E_{\mathbf{k}}=\sqrt{\Delta^{2}+\epsilon_{\mathbf{k}}^{2}}$ is the AF band energy, and $\Delta$ is obtained from the self-consistency condition $(1 / N) \Sigma_{\mathbf{k}}\left(2 E_{\mathbf{k}}\right)^{-1}=U^{-1}$.

Now, for a given disorder strength $W$, the inverse potential (absolute value) has a lower bound $1 /\left|\epsilon_{i}\right|>2 / W$. Therefore poles in the $T$ matrix are present for $\left|\left[g_{\sigma}^{0}\right]_{i i}\right|>2 / W$, so that disorder-induced states are formed within the gap, as shown by hatched regions in Fig. 1 . If $-\widetilde{\Delta}$ and $\widetilde{\Delta}$ mark the energies (shown by arrows) up to which states are formed, then $\widetilde{\Delta}$ is obtained from $\left[g_{\sigma}^{0}(-\widetilde{\Delta})\right]_{i i}=2 / W$. The remaining Hubbard gap $2 \widetilde{\Delta}$ is thus obtained from the solution of

$$
\frac{1}{N} \sum_{\mathbf{k}} \frac{\Delta+\widetilde{\Delta}}{E_{\mathbf{k}}^{2}-\widetilde{\Delta}^{2}}=\frac{2}{W} .
$$

A plot of the normalized energy gap $2 \widetilde{\Delta} / 2 \Delta$ is shown in Fig. 2 as a function of the relative disorder strength $W / U$ for $U / t=10$, indicating an almost linear reduction with disorder strength. 


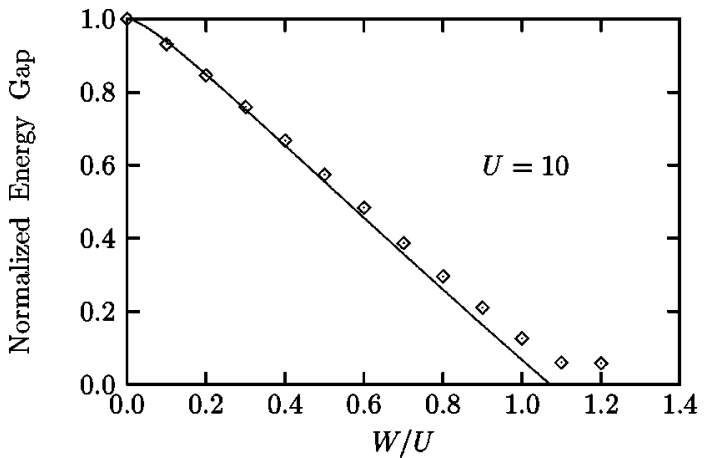

FIG. 2. Normalized energy gap vs $W / U$ at $T=0$, from the $T$-matrix analysis (line), and the numerical UHF analysis for a 10 $\times 10$ lattice (squares).

With increasing disorder strength states are formed deeper in the Hubbard gap, and when states have approached from both sides in the middle of the Hubbard gap, the energy gap vanishes. The critical disorder strength $W_{c}$ at which the energy gap $2 \tilde{\Delta}$ vanishes is therefore given by $\left[g_{\sigma}^{0}(0)\right]_{i i}$ $=2 / W_{c}$, which yields the following equation for the critical disorder strength:

$$
\frac{1}{N} \sum_{\mathbf{k}} \frac{\Delta}{\Delta^{2}+\epsilon_{\mathbf{k}}^{2}}=\frac{2}{W_{c}}
$$

Considering the strong-correlation limit as a special case, and keeping terms up to order $t^{2} / U^{2}$, the critical disorder strength is then given by $W_{c} / U=1+8 t^{2} / U^{2}$, where we used $m=1-8 t^{2} / U^{2}$ and $(1 / N) \Sigma_{\mathbf{k}} \epsilon_{\mathbf{k}}^{2}=4 t^{2}$ for the square lattice. Thus, with decreasing interaction strength, the ratio $W_{c} / U$ actually increases. This is because the kinetic energy becomes relatively important with decreasing interaction strength, and the bandwidth starts competing with disorder strength.

\section{B. Numerical UHF analysis}

In order to check the validity of the $T$-matrix approximation, we have also used a numerical UHF analysis. In this approach, the HF Hamiltonian on a finite lattice is numerically diagonalized self-consistently, so that disorder is treated exactly. This approach has been described earlier in the context of hole/impurity doping in the Hubbard antiferromagnet. ${ }^{46,47}$ The energy gap is obtained from the energy difference between the lowest-energy state of the upper Hubbard band and the highest-energy state of the lower Hubbard band. Configuration averaging is performed over 100 different realizations of the random on-site potentials on a $10 \times 10$ lattice. The reduction in energy gap with disorder strength using this method is also shown in Fig. 2. The numerical analysis shows a saturation of the energy difference at $W / U \sim 1$ due to finite system size. Deviations from the $T$-matrix approach are more pronounced at lower interaction strengths where the fermion states are more extended. The almost linear reduction of energy gap with disorder is also seen at finite temperatures, as shown in Fig. 3. The critical disorder strength decreases rapidly with increasing temperature.

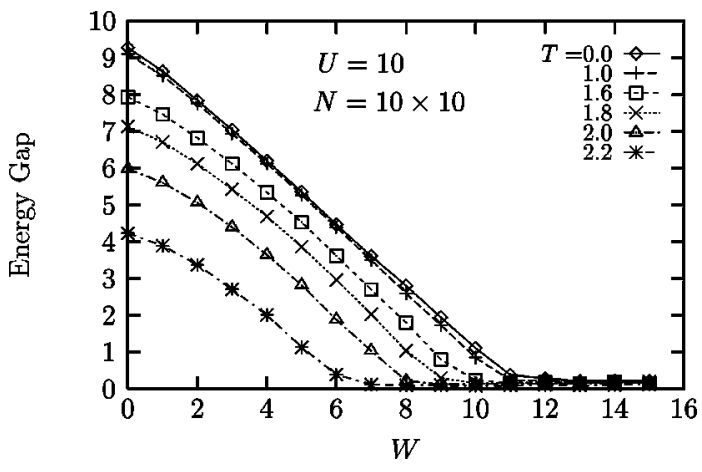

FIG. 3. Energy gap vs $W$ from the numerical UHF analysis at different temperatures (shown against plotting symbols).

\section{MAGNON RENORMALIZATION}

Magnons are the low-energy excitations associated with transverse spin fluctuations in the broken-symmetry state of systems possessing continuous spin-rotational symmetry. Therefore they play an important role in several macroscopic properties such as the temperature dependence of the order parameter, Néel temperature, specific heat, etc. We therefore consider the magnon propagator and obtain the disorderinduced renormalizations in magnon energies, transverse spin correlations, and the quantum spin-fluctuation correction to sublattice magnetization. The magnon propagator with site indices $i, j$ is defined in terms of spin-lowering and spin-raising operators by $G_{i j}^{-+}$ $=\left\langle\Psi_{\mathrm{G}}\left|T\left[S_{i}^{-}(t) S_{j}^{+}\left(t^{\prime}\right)\right]\right| \Psi_{\mathrm{G}}\right\rangle$. We take the Green's-function approach and write the RPA result in $\omega$ space as

$$
G^{-+}=\frac{\chi_{\mathrm{dis}}^{0}}{1-U \chi_{\mathrm{dis}}^{0}}=\frac{G^{0}}{1-\Sigma G^{0}} .
$$

Here the matrix (in site indices) $\chi_{\text {dis }}^{0}$ is the zeroth-order antiparallel-spin particle-hole propagator for the disordered $\mathrm{AF}$, with matrix elements given by $\left[\chi_{\text {dis }}^{0}(\omega)\right]_{i j}$ $=i \int\left(d \omega^{\prime} / 2 \pi\right)\left[g_{\uparrow}\left(\omega^{\prime}\right)\right]_{i j}\left[g_{\downarrow}\left(\omega^{\prime}-\omega\right)\right]_{j i}$, written in terms of the one-particle Green's function $\left[g_{\sigma}(\omega)\right]_{i j}$, and evaluated in the self-consistent, broken-symmetry state. By $\chi_{\text {pure }}^{0}$ we define the corresponding quantity for the pure $\mathrm{AF}$, in terms of which the matrix $G^{0}=\chi_{\text {pure }}^{0} /\left(1-U \chi_{\text {pure }}^{0}\right)$ is the magnon propagator for the pure AF. Furthermore, the disorder selfenergy $\Sigma=U^{2} \delta \chi^{0}$ is expressed in terms of the disorderinduced perturbation $\delta \chi^{0} \equiv \chi_{\text {dis }}^{0}-\chi_{\text {pure }}^{0}$. Details of the perturbative analysis for $\delta \chi^{0}$ in the strong-coupling limit are given in the Appendix, and we use the result here for the disorder self-energy $\Sigma$ that has diagonal and nearest-neighbor (NN) terms. For the pure AF in the strong-coupling limit and in the two-sublattice basis (indices $A, B$ ), the propagator in $\mathbf{k}$ space takes the form ${ }^{46}$

$$
\left[G^{0}\right]^{-1}(\mathbf{k}, \omega)=\left[\begin{array}{ll}
1+\omega & \gamma_{\mathbf{k}} \\
\gamma_{\mathbf{k}} & 1-\omega
\end{array}\right],
$$

in energy units where $2 J=8 t^{2} / U=1$. Here $\gamma_{\mathbf{k}} \equiv\left(\cos k_{x}\right.$ $\left.+\cos k_{y}\right) / 2$, so that in real space $\left[G^{0}\right]^{-1}$ has only diagonal elements, $\left[G^{0}\right]_{i i}^{-1}=1 \pm \omega$ for site $i$ in $A$ and $B$ sublattices, and NN matrix elements $\left[G^{0}\right]_{i, i+\delta}^{-1}=1 / Z$, where $i+\delta$ refers to $\mathrm{NN}$ of $i$. 
We first consider the configuration-averaged self-energy $\langle\Sigma\rangle=U^{2}\left\langle\delta \chi^{0}\right\rangle$. This approximation neglects vertex corrections that, however, appear only at order $(W / U)^{4}$, and are discussed separately in the next section. From the results given in the Appendix for the matrix elements of $\delta \chi^{0}$ up to order $(W / U)^{2}$, we obtain $\left\langle\Sigma_{i i}\right\rangle=-\sigma$ and $\left\langle\Sigma_{i, i+\delta}\right\rangle$ $=-\sigma / Z$, in units of $U^{2} t^{2} / \Delta^{3}$, where $\sigma \equiv(1 / 6) W^{2} / U^{2}$. Substituting the translationally symmetric $\langle\Sigma\rangle$ in Eq. (4), Fourier transforming $\left[G^{0}\right]^{-1}-\langle\Sigma\rangle$, and finally inverting, we obtain the following expression for the renormalized magnon propagator in $\mathbf{k}$ space and the two-sublattice basis:

$$
\begin{aligned}
G^{-+}(\mathbf{k}, \omega)= & -\frac{1}{2} \frac{1}{\sqrt{1-\gamma_{\mathbf{k}}^{2}}}\left[\begin{array}{cc}
1-\frac{\omega}{2 \widetilde{J}} & -\gamma_{\mathbf{k}} \\
-\gamma_{\mathbf{k}} & 1+\frac{\omega}{2 \widetilde{J}}
\end{array}\right] \\
& \times\left(\frac{1}{\omega-\tilde{\omega}_{\mathbf{k}}+i \eta}-\frac{1}{\omega+\tilde{\omega}_{\mathbf{k}}-i \eta}\right),
\end{aligned}
$$

containing both the retarded and advanced parts having poles below and above the real- $\omega$ axis, respectively. Here $\widetilde{J} \equiv J(1$ $+\sigma)$, and $\tilde{\omega}_{\mathbf{k}}=2 \widetilde{J} \sqrt{1-\gamma_{\mathbf{k}}^{2}}$ is the disorder-renormalized magnon energy. Thus at $\mathcal{O}(W / U)^{2}$ the form of the magnon propagator remains unchanged, and there is only a momentum-independent multiplicative renormalization of magnon energies, leading to an effective stiffening of the magnon modes by disorder. An upward shift of the magnon band in the strong-correlation and weak-disorder limit has also been observed in a numerical RPA study in three dimensions. $^{43}$

This effective enhancement of the magnon energy scale can also be viewed as resulting from the enhancement in the configuration-averaged $\mathrm{NN}$ exchange energy $\left\langle t^{2} /\left(U+\epsilon_{i}\right.\right.$ $\left.\left.-\epsilon_{j}\right)\right\rangle$ which, to second order in $W / U$, is $(1+\sigma) t^{2} / U$. To the extent that the finite-temperature reduction in sublattice magnetization due to thermal excitation of magnons is suppressed by this enhancement, the disordered AF exhibits a slower $m(T)$ vs $T$ falloff, and therefore a higher Néel temperature (for $D>2$ ). For strong coupling, the Néel state is therefore stabilized by weak disorder, as also reported in the DMFT (Ref. 40) and the Onsager-reaction-field ${ }^{44}$ studies.

Quantum correction to sublattice magnetization. As only the effective exchange energy scale gets modified in Eq. (6), while the form is not changed by disorder ${ }^{46}$ the magnitude of equal-time, same-site transverse spin correlations $\left\langle S_{i}^{-} S_{i}^{+}\right\rangle$ and $\left\langle S_{i}^{+} S_{i}^{-}\right\rangle$remain unchanged. These transverse spin correlations are obtained by frequency integration of the diagonal elements of transverse spin propagators, $\left\langle S_{i}^{-}(t) S_{i}^{+}\left(t^{\prime}\right)\right\rangle$ $=-i \int(d \omega / 2 \pi)\left[G^{-+}(\omega)\right]_{i i} \exp \left\{-i w\left(t-t^{\prime}\right)\right\}$, where the appropriate part (retarded or advanced) of $G^{-+}$is taken depending on whether $t^{\prime}<t$ or $t^{\prime}>t$. Taking $t^{\prime} \rightarrow t^{-}$, and using the retarded part of $G^{-+}$from Eq. (6), we obtain

$$
\left\langle S_{i}^{-} S_{i}^{+}\right\rangle=\frac{1}{2} \frac{1}{N} \sum_{\mathbf{k}}\left[\frac{1}{\sqrt{1-\gamma_{\mathbf{k}}^{2}}}-1\right],
$$

$$
\left\langle S_{i}^{+} S_{i}^{-}\right\rangle=\frac{1}{2} \frac{1}{N} \sum_{\mathbf{k}}\left[\frac{1}{\sqrt{1-\gamma_{\mathbf{k}}^{2}}}+1\right] .
$$

Here the second result is obtained using the relationship $G_{A A}^{+-}(\omega)=G_{B B}^{-+}(\omega)$ that follows from spin-sublattice symmetry. The result for total transverse spin fluctuation $\left\langle S_{i}^{-} S_{i}^{+}+S_{i}^{+} S_{i}^{-}\right\rangle$, is thus identical to the RPA result for the pure Hubbard $\mathrm{AF}^{45}{ }^{45}$ as well as the spin-wave-theory (SWT) result for the quantum Heisenberg antiferromagnet $(\mathrm{QHAF}){ }^{48,49}$ Therefore up to order $(W / U)^{2}$ the quantum spin-fluctuation correction to sublattice magnetization in the strong-coupling limit remains unchanged from the spinwave-theory result $\delta m_{\mathrm{SF}}=(1 / N) \Sigma_{\mathbf{k}}\left[\left(1-\gamma_{\mathbf{k}}^{2}\right)^{-1 / 2}-1\right] \approx 0.39$ in two dimensions. ${ }^{48,49}$

\section{VERTEX CORRECTIONS AND MAGNON DAMPING}

In this section we consider vertex corrections that were neglected in the previous section due to use of the configuration-averaged self-energy $\langle\Sigma\rangle$ in Eq. (4). As at higher orders this approximation produces terms like $G^{0}\langle\Sigma\rangle G^{0}\langle\Sigma\rangle G^{0}$ in Eq. (4); we therefore subtract out this term and focus here on the configuration-averaged proper self-energy at second order,

$$
\Gamma=\left\langle\Sigma G^{0} \Sigma\right\rangle-\langle\Sigma\rangle G^{0}\langle\Sigma\rangle
$$

which precisely incorporates the vertex corrections. As shown later in this section, this second-order scattering process results in magnon damping, which therefore only appears at order $(W / U)^{4}$. We note here that the term "magnon damping" in this section refers to the decay of a momentum mode, and not to the decay into particle-hole excitations, which is energetically ruled out in the strong-correlation limit where $J \ll U$.

Considering the matrix element $\Gamma_{i j}$, we note that since $\Sigma$ is only limited to diagonal and NN matrix elements, if sites $i$ and $j$ are far apart, then there are no correlations between the two $\Sigma$ terms, and the difference vanishes. Therefore the proper self-energy $\Gamma$ arises only from local correlations in the disorder self-energy terms, and has diagonal, NN, and next-nearest-neighbor (NNN) matrix elements. The vertex corrections therefore result in new NNN spin couplings in the magnon propagator, besides renormalizing the NN couplings. Such longer-range spin couplings also arise in the Hubbard AF at intermediate and weak couplings.

For the square lattice $(Z=4)$, we give below the results for matrix elements of $\Gamma$ for the case $i$ in $A$ sublattice ( $i$ $\in A)$, results for the other case following from symmetry. Also given are the results after substitution of the matrix elements of $G^{0}(\mathbf{k}, \omega)$, obtained from Eq. (5). We now illustrate the evaluation of $\Gamma$ for the diagonal-matrix element. Expanding the matrix product, and using the property of the disorder self-energy that the diagonal element $\Sigma_{i i}$ equals the sum of the NN elements $\Sigma_{i, i+\delta}$, this can be written as $\Gamma_{i i}=\left[\left(\left\langle\Sigma_{i, i+\delta} \Sigma_{i+\delta^{\prime}, i}\right\rangle-\left\langle\Sigma_{i, i+\delta}\right\rangle\left\langle\Sigma_{i+\delta^{\prime}, i}\right\rangle\right)\left(G_{i i}^{0}+G_{i+\delta, i}^{0}\right.\right.$ $\left.\left.+G_{i, i+\delta^{\prime}}^{0}+G_{i+\delta, i+\delta^{\prime}}^{0}\right)\right]$, where summation over $\delta$ and $\delta^{\prime}$ is implied. Configuration averaging, with $\sigma_{2}$ $\equiv\left\langle\epsilon_{i}^{2}\right\rangle / U^{2}$ and $\sigma_{4} \equiv\left\langle\epsilon_{i}^{4}\right\rangle / U^{4}$, for the second and fourth moments, yields $\quad\left(\left\langle\Sigma_{i, i+\delta} \Sigma_{i+\delta^{\prime}, i}\right\rangle-\left\langle\Sigma_{i, i+\delta}\right\rangle\left\langle\Sigma_{i+\delta^{\prime}, i}\right\rangle\right)$ $=Z^{-2}\left[\left(\sigma_{4}-\sigma_{2}^{2}\right)+\left(\sigma_{4}+3 \sigma_{2}^{2}\right) \delta_{\delta \delta^{\prime}}\right]$. Substituting $G_{i j}^{0}(\omega)$ 
$=(1 / N) \sum_{\mathbf{k}} G^{0}(\mathbf{k}, \omega) \exp \left\{i \mathbf{k} \cdot\left(\mathbf{r}_{i}-\mathbf{r}_{j}\right)\right\}$, and taking the appropriate matrix elements of $G^{0}(\mathbf{k}, \omega)$ in the two-sublattice basis, depending on sublattices of sites $i$ and $j$, yields the expression for $\Gamma_{i i}$ in Eq. (9). Similarly evaluating the NN and NNN elements, with $i+\delta, i+\kappa$, and $i+\kappa^{\prime}$ standing for the $\mathrm{NN}, \mathrm{NNN}$ (diagonal), and NNN (straight) of $i$, respectively, we obtain

$$
\begin{aligned}
\Gamma_{i i}=\left(\sigma_{4}-\sigma_{2}^{2}\right)\left[G_{A A}^{0}+\left(G_{A B}^{0}+G_{B A}^{0}\right) \gamma_{\mathbf{k}}+G_{B B}^{0} \gamma_{\mathbf{k}}^{2}\right] \\
+Z^{-1}\left(\sigma_{4}+3 \sigma_{2}^{2}\right)\left[G_{A A}^{0}+\left(G_{A B}^{0}+G_{B A}^{0}\right) \gamma_{\mathbf{k}}+G_{B B}^{0}\right] \\
=\left(\sigma_{4}-\sigma_{2}^{2}\right)\left[(1-\omega)\left(1-\gamma_{\mathbf{k}}^{2}\right) /\left(\omega_{\mathbf{k}}^{2}-\omega^{2}\right)\right] \\
+Z^{-1}\left(\sigma_{4}+3 \sigma_{2}^{2}\right)\left[2\left(1-\gamma_{\mathbf{k}}^{2}\right) /\left(\omega_{\mathbf{k}}^{2}-\omega^{2}\right)\right] \\
\Gamma_{i, i+\delta}=Z^{-1}\left(\sigma_{4}-\sigma_{2}^{2}\right)\left[\left(G_{A A}^{0}+G_{B B}^{0}\right)\left(1+\gamma_{\mathbf{k}}^{2}\right)\right. \\
\left.+2\left(G_{A B}^{0}+G_{B A}^{0}\right) \gamma_{\mathbf{k}}\right]+4 Z^{-2} \sigma_{2}^{2}\left[\left(G_{A A}^{0}+G_{B B}^{0}\right)\right. \\
\left.+\left(G_{A B}^{0}+G_{B A}^{0}\right) \gamma_{\mathbf{k}}\right] \\
=Z^{-1}\left(\sigma_{4}-\sigma_{2}^{2}\right)\left[2\left(1-\gamma_{\mathbf{k}}^{2}\right) /\left(\omega_{\mathbf{k}}^{2}-\omega^{2}\right)\right] \\
+4 Z^{-2} \sigma_{2}^{2}\left[2\left(1-\gamma_{\mathbf{k}}^{2}\right) /\left(\omega_{\mathbf{k}}^{2}-\omega^{2}\right)\right] \\
\Gamma_{i, i+\kappa}=Z^{-2}\left(\sigma_{4}-\sigma_{2}^{2}\right) 2\left[G_{A A}^{0} \cos _{x} \cos k_{y}\right. \\
\left.\quad+\left(G_{A B}^{0}+G_{B A}^{0}\right) \gamma_{\mathbf{k}}+G_{B B}^{0}\right] \\
=Z^{-2}\left(\sigma_{4}-\sigma_{2}^{2}\right) 2\left[\left\{2\left(1-\gamma_{\mathbf{k}}^{2}\right)-(1-\omega)\right.\right. \\
\left.\left.\quad \times\left(1-\cos k_{x} \cos k_{y}\right)\right\} /\left(\omega_{\mathbf{k}}^{2}-\omega^{2}\right)\right] \\
\Gamma_{i, i+\kappa^{\prime}}=Z^{-2}\left(\sigma_{4}-\sigma_{2}^{2}\right)\left[G_{A A}^{0} \gamma_{2 \mathbf{k}}+\left(G_{A B}^{0}+G_{B A}^{0}\right) \gamma_{\mathbf{k}}+G_{B B}^{0}\right] \\
=Z^{-2}\left(\sigma_{4}-\sigma_{2}^{2}\right)\left[\left\{2\left(1-\gamma_{\mathbf{k}}^{2}\right)\right.\right. \\
\left.\left.-(1-\omega)\left(1-\gamma_{2 \mathbf{k}}\right)\right\} /\left(\omega_{\mathbf{k}}^{2}-\omega^{2}\right)\right]
\end{aligned}
$$

Here the summation over momentum $\mathbf{k}$ is implied. A straightforward check confirms that the sum of matrix elements $\Gamma_{i i}+Z \Gamma_{i, i+\kappa}+Z \Gamma_{i, i+\kappa^{\prime}}$ involving sites of the same sublattice (diagonal and NNN) exactly equals the sum $Z \Gamma_{i, i+\delta}$ involving sites on opposite sublattices. This ensures that the Goldstone mode, which has amplitudes 1 and -1 on the two sublattice sites, is preserved, as expected from the continuous spin-rotational symmetry. We also notice that the various terms involve $k$ sums of the type $\Sigma k^{2} /\left(c^{2} k^{2}-\omega^{2}\right)$ from long-wavelength internal magnon modes. Therefore the self-energy terms are all nonsingular in two dimensions.

We now proceed with the magnon renormalization due to this proper self-energy correction $\Gamma$ up to order $W^{4}$. To this order, it is sufficient to examine the lowest-order correction $\langle\mathbf{q}|\Gamma| \mathbf{q}\rangle$ to the eigenvalue of the $U\left(1-U \chi_{\text {pure }}^{0}\right)$ matrix for the pure AF. The relevant eigenvalue is $1-\sqrt{\omega^{2}+\gamma_{\mathbf{q}}^{2}}$ in energy units such that $2 J=1$. The magnon amplitudes for state $|\mathbf{q}\rangle$ are $\sin \theta / 2$ and $-\cos \theta / 2$ in the two-sublattice basis, where $\cos \theta=\omega / \sqrt{\omega^{2}+\gamma_{\mathbf{q}}^{2}}$ and $\sin \theta=\gamma_{\mathbf{q}} / \sqrt{\omega^{2}+\gamma_{\mathbf{q}}^{2}}{ }^{21}$ For $\omega=\omega_{\mathbf{q}}$ $=\sqrt{1-\gamma_{\mathbf{q}}^{2}}$ the magnon energy, these amplitudes become $\sqrt{1-\omega_{\mathbf{q}}}$ and $-\sqrt{1+\omega_{\mathbf{q}}}$, respectively. Using the matrix elements of $\Gamma$ from above we obtain for the eigenvalue correction $\delta \lambda_{\mathbf{q}}^{(2)}=\langle\mathbf{q}|\Gamma| \mathbf{q}\rangle$,

$$
\begin{aligned}
\delta \lambda_{\mathbf{q}}^{(2)}= & \frac{2}{N} \sum_{i \in A}\left[\operatorname { s i n } ^ { 2 } ( \theta / 2 ) \left(\Gamma_{i i}+\Gamma_{i, i+\kappa} Z \cos q_{x} \cos q_{y}\right.\right. \\
& \left.\left.+\Gamma_{i, i+\kappa^{\prime}} Z \gamma_{2 \mathbf{q}}\right)-\sin (\theta / 2) \cos (\theta / 2) \Gamma_{i, i+\delta} Z \gamma_{\mathbf{q}}\right] \\
& +\frac{2}{N} \sum_{j \in B}\left[\operatorname { c o s } ^ { 2 } ( \theta / 2 ) \left(\Gamma_{j j}+\Gamma_{j, j+\kappa} Z \cos q_{x} \cos q_{y}\right.\right. \\
& \left.\left.+\Gamma_{j, j+\kappa^{\prime}} Z \gamma_{2 \mathbf{q}}\right)-\cos (\theta / 2) \sin (\theta / 2) \Gamma_{j, j+\delta} Z \gamma_{\mathbf{q}}\right] .
\end{aligned}
$$

In the second term above (for sites $j$ in the $B$ sublattice) the matrix elements of $\Gamma$ follow from Eq. (9) with $\omega$ replaced by $-\omega$, in view of Eq. (5). The magnon energy for mode $\mathbf{q}$ is then given by the solution of $1-\sqrt{w^{2}+\gamma_{\mathbf{q}}^{2}}-\delta \lambda_{\mathbf{q}}^{(1)}$ $-\delta \lambda_{\mathbf{q}}^{(2)}(\omega)=0$. Here $\delta \lambda_{\mathbf{q}}^{(1)}=-\sigma\left(1-\gamma_{\mathbf{q}}^{2}\right)$ is the eigenvalue correction due to the first-order self-energy $\langle\Sigma\rangle$; its effect on magnon stiffening has been discussed earlier.

We first consider the magnon renormalization in the longwavelength $(q \ll 1)$, low-energy $(\omega \ll 1)$ limit for simplicity. We can drop $\omega$ in the numerators in Eq. (9) for the selfenergy $\Gamma$, which removes the sublattice dependence, and the above eigenvalue correction simplifies in this limit to

$$
\begin{aligned}
\delta \lambda_{\mathbf{q}}^{(2)}(\omega \ll 1)= & {\left[\Gamma_{i i}+\Gamma_{i, i+\kappa} Z \cos q_{x} \cos q_{y}\right.} \\
& \left.+\Gamma_{i, i+\kappa^{\prime}} Z \gamma_{2 \mathbf{q}}-\Gamma_{i, i+\delta} Z \gamma_{\mathbf{q}} \sin \theta\right] \\
= & Z\left[\Gamma_{i, i+\delta}\left(1-\gamma_{\mathbf{q}} \sin \theta\right)\right. \\
& -\Gamma_{i, i+\kappa}\left(1-\cos q_{x} \cos q_{y}\right) \\
& \left.-\Gamma_{i, i+\kappa^{\prime}}\left(1-\gamma_{2 \mathbf{q}}\right)\right] \\
\approx & \alpha q^{2} .
\end{aligned}
$$

As expected the eigenvalue correction goes like $q^{2}$. Here the identity $\Gamma_{i i}=Z\left[\Gamma_{i, i+\delta}-\Gamma_{i, i+\kappa}-\Gamma_{i, i+\kappa^{\prime}}\right]$, which ensures the preservation of the Goldstone mode has been used, and the coefficient $\alpha$ is given by

$$
\alpha=Z\left(\frac{\Gamma_{i, i+\delta}}{4}-\frac{\Gamma_{i, i+\kappa}}{2}-\Gamma_{i, i+\kappa^{\prime}}\right) .
$$

Considering now the case of a general $\omega$, we find that the following terms are present in addition to those given in Eq. (11):

$$
\begin{aligned}
\delta \lambda_{\mathbf{q}}^{(2)}-\delta \lambda_{\mathbf{q}}^{(2)}(\omega \ll 1)= & \omega \cos \theta(1 / N) \sum_{\mathbf{k}} \\
& \times\left[\left(\sigma_{4}-\sigma_{2}^{2}\right)\left(1-\gamma_{\mathbf{k}}^{2}\right) /\left(\omega_{\mathbf{k}}^{2}-\omega^{2}\right)\right. \\
& -2 Z^{-1}\left(\sigma_{4}-\sigma_{2}^{2}\right) \cos q_{x} \cos q_{y} \\
& \times\left(1-\cos k_{x} \cos k_{y}\right) /\left(\omega_{\mathbf{k}}^{2}-\omega^{2}\right) \\
& -Z^{-1}\left(\sigma_{4}-\sigma_{2}^{2}\right) \gamma_{2 \mathbf{q}}\left(1-\gamma_{2 \mathbf{k}}\right) /\left(\omega_{\mathbf{k}}^{2}\right. \\
& \left.\left.-\omega^{2}\right)\right] .
\end{aligned}
$$

We now focus on the imaginary part of this second-order correction $\delta \lambda_{\mathbf{q}}^{(2)}$. For this purpose we examine the internal momentum sums of the type $(1 / N) \Sigma_{\mathbf{k}} 2 \omega_{\mathbf{k}}^{2} /\left(\omega_{\mathbf{k}}^{2}-\omega^{2}\right)$ that appear in the eigenvalue correction above. Using the following identity for the imaginary part, 


$$
\Im \frac{1}{N} \sum_{\mathbf{k}} \frac{2 \omega_{\mathbf{k}}^{2}}{\omega_{\mathbf{k}}^{2}-\omega^{2}}=\pi \frac{1}{N} \sum_{\mathbf{k}} \omega_{\mathbf{k}} \delta\left(\omega_{\mathbf{k}}-\omega\right)=\pi \omega N(\omega),
$$

for positive $\omega$, we obtain the imaginary part in terms of the magnon density of states $N(\omega)$. We consider the two limiting cases of low-energy magnon modes $\omega \ll 1$ and highenergy modes with $\omega \sim 1$. For long-wavelength, low-energy modes, $N(\omega) \sim \omega$, therefore $\Im \delta \lambda_{\mathbf{q}}^{(2)} \sim D q^{2} \omega^{2}$, where $D$ $=\Im \alpha / \omega^{2}$ in terms of the $q^{2}$ coefficient given in Eq. (12). We thus find the magnon energy to be given by

$$
\omega_{\mathbf{q}}=c q\left[1+i D q^{2}\right],
$$

where $c$ is the renormalized magnon velocity. The ratio $\Gamma_{\mathbf{q}} / \omega_{\mathbf{q}}$ of the magnon damping term to the energy thus vanishes like $q^{2}$ in the long-wavelength limit, indicating weak disorder scattering caused by the averaging-out of the on-site potential disorder at long length scales.

Long-wavelength magnon modes therefore continue to be well-defined excitations even with disorder. However, for short-wavelength, high-energy modes with energy $\omega \sim 1$, the presence in the imaginary term of the magnon density of states, which actually diverges (logarithmically in two dimensions) at the upper band edge at energy $2 J$, indicates that high-energy modes are strongly damped. A self-consistent evaluation is therefore required, with an imaginary term in the internal magnon mode propagator. This leads to a selfconsistent magnon damping of order $(W / U)^{4}$. In as much as the two-magnon Raman scattering process probes shortwavelength, high-energy magnon modes, this magnon damping of order $(W / U)^{4}$ will be important in an analysis of Raman linewidth in disordered antiferromagnets.

\section{DISORDER-ENHANCED DELOCALIZATION}

All the disorder effects in the AF state obtained so far can be understood within a single paradigm of disorderenhanced delocalization, i.e., an enhancement of the effective $t / U$ ratio due to disorder. Thus, the disorder-induced enhancement of magnon energy scale and Néel temperature in strong coupling, as well as the reduction of sublattice magnetization (discussed in the Appendix), can be viewed as arising from this enhancement of the effective $t / U$ ratio. This delocalization effect of disorder, contrary to its usual tendency to localize, is characteristic of the AF state with its inherent localization due to Coulomb barriers, and can be understood as follows. A positive on-site energy $\epsilon_{i}$ reduces the potential barrier for the majority-spin electron, which enhances its probability for tunneling through, thereby lowering the on-site density. On the other hand, a negative on-site energy increases the potential barrier, which has a weaker effect if the electron is already localized. Thus the effects of positive and negative on-site energies are asymmetrical, leading to enhanced delocalization on the average. This asymmetrical effect is clearly seen in Fig. 4 showing the electronic densities for different on-site energies, obtained within the DMFT.

This disorder-enhanced delocalization also qualitatively accounts for opposite disorder effects on $T_{N}$ that are observed for strong and weak coupling. While for strong cou-

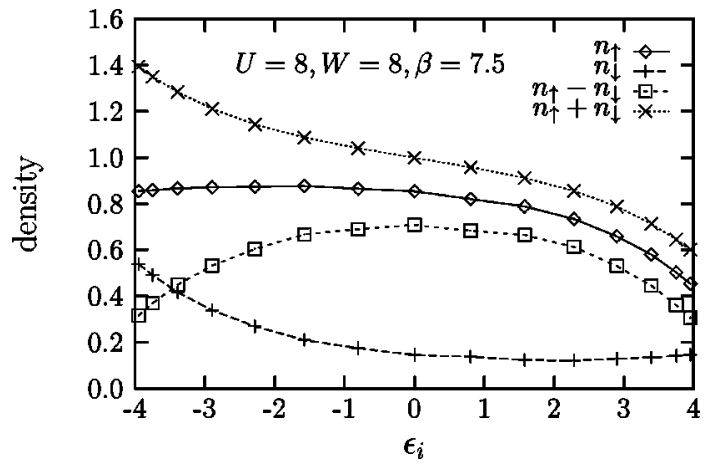

FIG. 4. Spin-dependent electronic densities on the $A$ sublattice vs on-site energy in the low-temperature ordered state as obtained within the DMFT. The bandwidth is chosen $B=8$, as in $d=2$.

pling, disorder enhances $T_{N}$ and stabilizes the Néel state, the behavior is reversed for weak coupling. ${ }^{40,44}$ Both behaviors can be understood in terms of an effective $U / t$ ratio that decreases with disorder. This is because in the pure Hubbard antiferromagnet $T_{N}$ vs $U / t$ actually goes through a maximum, so that with decreasing $U / t, T_{N}$ is either enhanced or suppressed depending on whether one is on the strong- or weak-coupling side of the peak.

Similar effects are also seen in the magnon velocity, $c$ $=\omega_{\mathbf{q}} / q$ in the limit $q \rightarrow 0$, which also shows a peak structure. ${ }^{46}$ For this purpose we have considered the lowenergy magnon modes on a $10 \times 10$ lattice. Magnon energies in the RPA are obtained by exact diagonalization of the $\chi^{0}(\omega)$ matrix, evaluated in the self-consistent state using the numerical UHF approach. ${ }^{46}$ The low-energy modes are fourfold degenerate for the pure $\mathrm{AF}$ on a square lattice, and therefore the normalized magnon velocity is obtained by averaging $\omega_{n}(W) / \omega_{n}(0)$ over the four lowest-energy modes. The normalized magnon velocity, configuration averaged over 20 different configurations, is shown in Fig. 5, for several values of $U / t$. For strong coupling, the magnon velocity increases with disorder, almost quadratically, in agreement with the perturbative result from Eq. (6). The behavior is reversed for weak coupling, and the magnon velocity decreases with disorder strength. As the temperature dependence of the sublattice magnetization in the low-temperature limit depends only on the energy scale of low-energy magnon modes, this also implies slower (faster) temperature falloff of $m$ in the strong- (weak-) coupling limit.

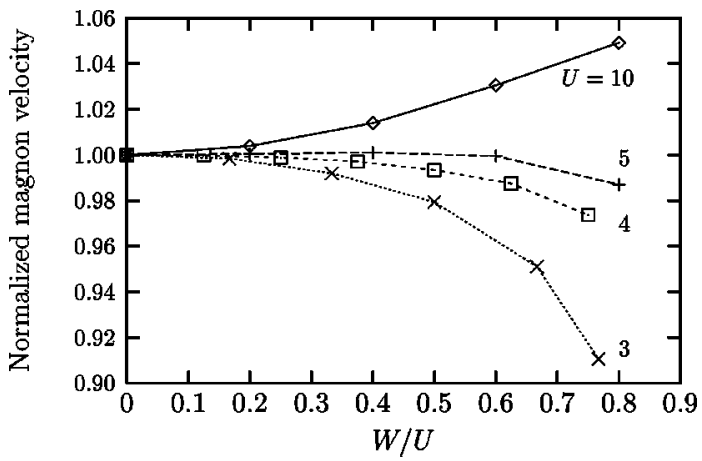

FIG. 5. Normalized magnon velocity $c(W) / c(0)$ vs $W / U$ for several interaction strengths, obtained from the low-energy magnon modes on a $10 \times 10$ lattice. 


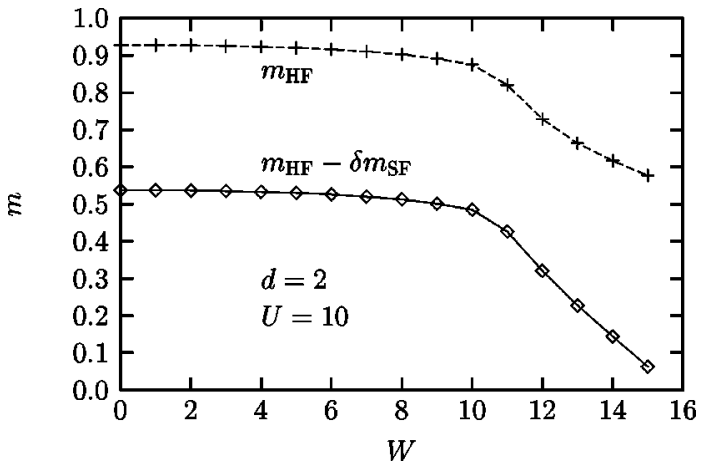

FIG. 6. Sublattice magnetization $m$ vs $W$ at the HF level for a $10 \times 10$ lattice (dashed line), and including spin fluctuations (solid line).

\section{STRONG DISORDER $(W>U)$ AND CROSSOVER BEHAVIOR}

We have seen earlier that with increasing disorder strength, the energy gap decreases almost linearly with $W$, and eventually closes when $W \sim U$. Therefore for $W>U$, assuming a single-occupancy constraint, the two Hubbard bands would overlap, indicating that single occupancy for all sites is no longer energetically favorable. Electrons from the highest-energy sites (with $\epsilon_{i}>U / 2$ ) are transferred to the lowest-energy sites (with $\epsilon_{i}<-U / 2$ ), making them doubly occupied and shifting up the energy by $U$. The electronic states associated with these essentially empty and doubly occupied sites are therefore located near the Fermi energy at $U / 2$. As these unoccupied and doubly occupied sites are nonmagnetic, the sublattice magnetization starts falling relatively rapidly with disorder strength. In the limit $t \ll U, W$ the bands are nearly flat with bandwidth $\sim W$, and the overlap region is $\sim(W-U)$, so that a simple estimate for the fraction of these nonmagnetic sites yields

$$
x \sim \frac{W-U}{W},
$$

indicating an almost linear decrease in sublattice magnetization for $W \sim U$. Thus a crossover takes place at $W \sim U$ from the essentially flat sublattice magnetization to an almost linear falloff with disorder. This is clearly seen in Fig. 6 where the configuration-averaged sublattice magnetization obtained within the UHF approximation is plotted against the disorder strength $W$.

However, a more significant consequence of the formation of these nonmagnetic sites is that they essentially act like spin vacancies in the antiferromagnet, which leads to spin-dilution behavior, as discussed in the following subsection. The above picture suggests that for $W>U$ the system can be viewed as a composite of a disordered AF with $\widetilde{W}$ $=U$, and a spin-diluted system with a concentration $x=(W$ $-U) / W$ of spin vacancies. Strong magnon scattering off static vacancies, leading to substantial softening of lowenergy, long-wavelength modes and magnon damping has been obtained earlier. ${ }^{16,18,21}$ The ratio of magnon damping term to its energy now goes like $(W-U) / W$, to be contrasted with the small damping ratio $(W / U)^{4} q^{2}$ in the weakdisorder regime, obtained earlier in Sec. IV. Vacancy- induced enhancement in transverse spin fluctuation has not been studied, and we discuss this in the following subsection.

\section{A. Enhanced fluctuations due to spin vacancies}

We present a simple estimate of the enhancement of transverse spin fluctuations due to spin vacancies. We consider the following Hubbard Hamiltonian on a square lattice with binary-distributed, random NN hopping:

$$
H=-\sum_{<i j>\sigma} t_{i j}\left(\hat{a}_{i \sigma}^{\dagger} \hat{a}_{j \sigma}+\text { H.c. }\right)+U \sum_{i} \hat{n}_{i \uparrow} \hat{n}_{i \downarrow},
$$

where the hopping term $t_{i j}=0$ if sites $i$ or $j$ are vacancy sites, and $t_{i j}=t$ otherwise. Thus for a vacancy on site $i$, all hopping terms $t_{i, i+\delta}$ connecting $i$ to its NN sites $i+\delta$ are set to zero. The vacancy site is thus completely decoupled from the system. Half-filling is retained by having one fermion per remaining site. We consider the $U / t \rightarrow \infty$ limit, where the local moments are fully saturated, and the model maps to the localized-spin Heisenberg model. In this limit the vacancy problem becomes identical to the spin vacancy problem in the QHAF, for which magnon renormalization was studied earlier. $^{16}$

The structure of the $\chi^{0}(\omega)$ matrix in the host AF, and the modification introduced by spin vacancies has been considered earlier in the context of static impurities. ${ }^{21}$ Since the vacancy spin is completely decoupled from the system, the magnitude of the diagonal-matrix element $\left[\chi^{0}\right]_{i i}$ on the vacancy site $i$ is irrelevant. To minimize the perturbation, we treat the vacancy site as occupied with an isolated spin. For a vacancy on site $i$, a perturbation is induced in the neighborhood due to the absence of hopping between vacancy site and NN sites $i+\delta$. In terms of the notation used in Sec. III, the following self-energy correction $\Sigma=U^{2} \delta \chi^{0}$ is obtained:

$$
\begin{gathered}
\Sigma_{i i}=1, \\
\Sigma_{i, i+\delta}=\Sigma_{i+\delta, i}=\Sigma_{i+\delta, i+\delta}=1 / 4,
\end{gathered}
$$

where again $2 J$ has been set to 1 . Thus, a diagonal contribution $\Sigma_{i i}$ arises if a vacancy exists either on site $i$, or on any of the four NN sites $i+\delta$. For a finite vacancy concentration $x$, the probability that a vacancy exists on a site is $x$. Therefore, configuration averaging yields $\left\langle\Sigma_{i i}\right\rangle=2 x$, and $\left\langle\Sigma_{i, i+\delta}\right\rangle=2 x / 4$. From Eq. (4) we obtain the same expression for the magnon propagator as in Eq. (6), except that now $\widetilde{J}$ $\equiv J(1-2 x)$, so that $\tilde{\omega}_{\mathbf{k}}=2 J(1-2 x) \sqrt{1-\gamma_{\mathbf{k}}^{2}}$ yields a softening of the magnon mode, reflecting the spin-dilution behavior. ${ }^{50}$ This magnon softening is in contrast to the stiffening in the weak-disorder regime, obtained earlier in Sec. III. Enhanced thermal excitation of magnons due to this softening will result in a faster temperature falloff of the sublattice magnetization, and hence to a lowering of the Néel temperature.

Since the form of the magnon propagator is not changed, as already discussed in Sec. III, the transverse spin correlations $\left\langle S_{i}^{-} S_{i}^{+}\right\rangle$and $\left\langle S_{i}^{+} S_{i}^{-}\right\rangle$, as well as the lattice-averaged spin-fluctuation correction to sublattice magnetization remain unchanged. Therefore, from the SWT result given after 


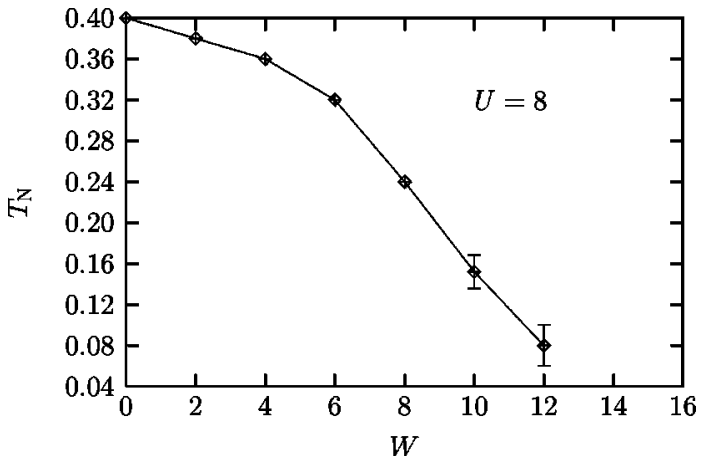

FIG. 7. Néel temperature $T_{N}$ vs $W$ obtained within the DMFT.

Eq. (7), $\delta m_{\mathrm{SF}} \approx 0.39$ in two dimensions. However, as the number of spins is reduced to $1-x$, the quantum correction per spin is enhanced.

In a recent numerical, finite-size study of the spinvacancy problem, ${ }^{51}$ focusing on the exact evaluation of transverse spin fluctuations in the RPA, the $x$ dependence of the quantum correction per spin $\delta m_{\mathrm{SF}} /(1-x)$ was found to be best described by the expression $0.39+0.42 x+6.5 x^{3}$. This results in a nearly vanishing $\mathcal{O}(x)$ term in $\delta m_{\mathrm{SF}}$, in agreement with the perturbative result obtained above. The lattice-averaged sublattice magnetization for the disordered $\mathrm{AF}$, obtained by accounting for the quantum spin-fluctuation correction using $m=m_{\mathrm{HF}}-\delta m_{\mathrm{SF}}$, with $\delta m_{\mathrm{SF}}=(1-x)(0.39$ $\left.+0.42 x+6.5 x^{3}\right)$, where the vacancy concentration $x=(W$ $-U) / W$ for $W>U$, is compared with the HF result in Fig. 6. The crossover at $W \sim U$ is again clearly seen from the rapid decrease in sublattice magnetization.

\section{B. Gapless antiferromagnetic state}

The above result indicates that substantial AF ordering remains even for $W \sim U$, where the energy gap closes. Since AF order persists for $W>U$ the gapless AF state is apparently quite robust even in two dimensions. This feature was also observed in the quantum Monte Carlo study in $d=2,{ }^{41}$ where substantial AF correlations were seen for $W=U$ $=4 t$, while the compressibility indicated an absence of the charge gap. This leads to the possibility, in three dimensions, of a metallic AF state, if states at Fermi energy are not localized. Gapless AF states, both metallic and insulating, were also obtained for the three-dimensional disordered Hubbard model, the phase diagram of which has been recently studied within the UHF approach. ${ }^{42}$ A region of metallic AF state in $d=3$ was also recently identified in the $t-t^{\prime}$ Hubbard model without disorder; here it is the NNN hopping amplitude $t^{\prime}$ that leads to frustration of AF order. ${ }^{52}$

The robustness of the gapless AF state is also seen from a DMFT calculation of the Néel temperature $T_{N}$, for a rectangular distribution of $\epsilon_{i}$, as obtained from an extrapolation to zero of the inverse AF susceptibility. Both exact enumeration and Monte Carlo evaluation were employed. Details of the application of DMFT to the disordered Hubbard model have been described earlier, ${ }^{40}$ where binary alloy and semicircular distributions were studied. The variation of $T_{N}$ with $W$ is shown in Fig. 7 for $U / B=1$. This corresponds to $U / t=8$ in the two-dimensional case. Appreciable spin ordering is evident from the fairly high $T_{N}$ even at $W \sim U$ where the energy

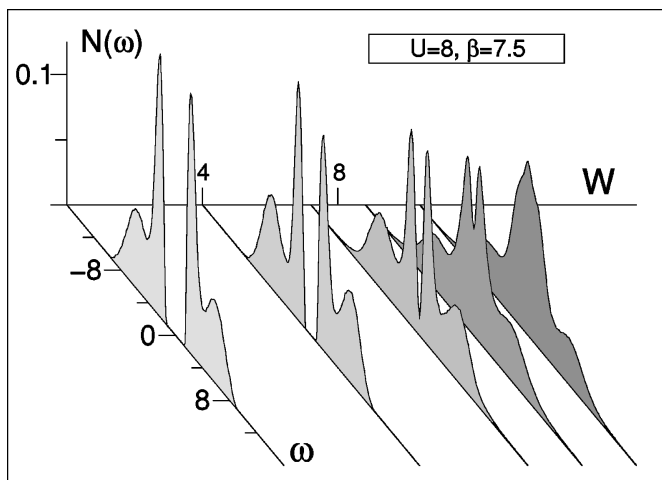

FIG. 8. Electronic density of states $N(\omega)$ in the low-temperature ordered state for several disorder values, as obtained within the DMFT. The charge gap closes near $W=U$.

gap vanishes. Within the DMFT, this closing of the energy gap with disorder, in the low-temperature ordered state is seen in Fig. 8 from the single-particle density of states $N(\omega)$, which was obtained by analytically continuing the imaginary-time Green's function using the maximum entropy procedure. ${ }^{53}$

As expected for weak and intermediate couplings, $T_{N}$ is seen to decrease with disorder strength, in contrast to the strong-coupling result of an enhancement in the magnon energy scale, and hence in $T_{N}$. Also, the critical disorder strength where $T_{N}$ vanishes is seen to be nearly $1.5 \mathrm{U}$, which is intermediate between the critical values of nearly $2 U$ for the milder semicircular distribution and nearly $U$ for the much more severe binary distribution. ${ }^{40}$ In fact, for the binary alloy case, the zero-temperature transition is expected to occur at $W \approx U$, when all sites abruptly become either unoccupied or doubly occupied and the sublattice magnetization vanishes.

\section{CONCLUSION AND DISCUSSION}

Two fundamentally different mechanisms-disorderenhanced delocalization and local-moment quenchingwere identified to control the magnetic behavior of an AF in the regimes of weak and strong disorder, respectively. In the weak disorder regime $(W<U)$ disorder effects on sublattice magnetization, magnon-mode energies, and Néel temperature can be qualitatively understood within the disorderenhanced delocalization effect. The stabilization of the Néel state by disorder in the strong-correlation limit, reflected in an enhancement of the magnon energy scale and the Néel temperature, is a striking consequence. In this regime the AF state is remarkably robust against disorder, particularly in the strong-correlation limit. Low-energy, long-wavelength magnon modes are weakly damped, and continue to be welldefined excitations.

With increasing disorder strength there is a crossover at $W \sim U$, characterized by a rapid decrease in sublattice magnetization and quenching of local moments due to formation of nonmagnetic sites. Driven by band overlap, this localmoment quenching can be viewed as the reverse of localmoment formation in the disordered metallic state with increasing $U / W$. These nonmagnetic sites act like spin vacancies in the antiferromagnet, leading to characteristic 
spin-dilution behavior. Vacancy-induced magnon scattering results in enhanced transverse spin fluctuations, softened magnon modes, lowering of Néel temperature, and strong magnon damping.

As discussed in Sec. VI, in the gapless AF state for $W$ $>U$, the electronic states near the Fermi energy are associated with (and localized around) the essentially empty and doubly occupied sites. These states are nonmagnetic in that they do not contribute significantly to local moments. Therefore it is interesting to note that the low-frequency conductivity behavior involves these nonmagnetic states near the Fermi energy. Further investigation of the gapless AF state, focusing on the low-frequency conductivity behavior, magnon damping due to decay into particle-hole excitations, and an estimation of the critical disorder strength where AFLRO is destroyed, is presently in progress. ${ }^{54}$

\section{ACKNOWLEDGMENT}

One of us (A.S.) gratefully acknowledges support from the Alexander von Humboldt Foundation.

\section{APPENDIX: PERTURBATIVE ANALYSIS IN $W / U$ : STRONG-CORRELATION LIMIT}

In this Appendix we treat the disorder term $V \equiv \sum_{i \sigma} \epsilon_{i} \hat{n}_{i \sigma}$ in the Hamiltonian as a perturbation and obtain disorder corrections in the broken-symmetry state up to second order in $W / U$. With $g_{\sigma}^{0}$ representing the one-electron HF Green's function in the pure $\mathrm{AF}$, the corresponding Green's function for the disordered AF, $g_{\sigma}=g_{\sigma}^{0} /\left[1-V g_{\sigma}^{0}\right]$, then yields disorder corrections to electronic densities and sublattice magnetization. Similarly, the magnon renormalization within RPA is obtained from the correction $\delta \chi^{0} \equiv \chi_{\text {dis }}^{0}-\chi_{\text {pure }}^{0}$ in the zeroth-order antiparallel-spin particle-hole propagator defined below Eq. (4).

For analytic convenience we consider the strongcorrelation limit and retain terms of order $t^{2} / U^{2}$ only, at which level the pure AF is equivalent to the NN Heisenberg model. Up to this order the Green's function $\left[g_{\sigma}^{0}\right]_{i j}$ in site basis has only diagonal, nearest-neighbor (NN), and nextnearest-neighbor (NNN) matrix elements. Only the former two are actually required in the strong-coupling analysis, and are given below for spin up and site $i$ in the $A$ sublattice. Expressions for other spin and sublattice cases follow from the spin-sublattice symmetry. These Green's functions are easily obtained by starting with the atomic limit, where the bands are at energies $-\Delta$ and $\Delta$, with $2 \Delta=U$, and then obtaining corrections up to second order in the hopping term $t$. The diagonal and NN matrix elements of the time-ordered Green's function matrix $\left[g_{\sigma}^{0}\right]$, containing both the advanced (lower band) and retarded (upper band) parts are

$$
\begin{gathered}
{\left[g_{\uparrow}^{0}\right]_{i i}=\frac{1-t^{2} / \Delta^{2}}{\omega-(-\Delta)-i \eta}+\frac{t^{2} / \Delta^{2}}{\omega-\Delta+i \eta},} \\
{\left[g_{\uparrow}^{0}\right]_{i, i+\delta}=\frac{-t / 2 \Delta}{\omega-(-\Delta)-i \eta}+\frac{t / 2 \Delta}{\omega-\Delta+i \eta} .}
\end{gathered}
$$

We note here that the corrections to one-particle Green's functions involve renormalizations of (i) density (wave func- tion) and (ii) energy. Diagrams in which the potential scattering is interband (involving different Hubbard bands) result in transfer of spectral weight across the Hubbard bands, and therefore yield density changes. However, the intraband processes (involving all propagators from the same band) represent energy renormalization due to disorder potential, and do not contribute to any density change. In the particle-hole propagator $\chi^{0}$, while both energy and density renormalizations need to be considered, diagrams involving only energy renormalization do not contribute as both the particle and hole energies are shifted identically by the disorder potential.

\section{First order}

The first-order correction $g_{\sigma}^{0} V g_{\sigma}^{0}$ yields the following interband contribution to the local one-particle Green's function for spin up:

$$
\begin{aligned}
{\left.\left[\delta g_{\uparrow}^{(1)}\right]_{i i}\right|_{\text {interband }}=} & 2\left[\frac{1}{\omega-(-\Delta)-i \eta} \epsilon_{i} \frac{t^{2} / \Delta^{2}}{\omega-\Delta+i \eta}\right. \\
& \left.+\sum_{\delta} \frac{-t / 2 \Delta}{\omega-(-\Delta)-i \eta} \epsilon_{i+\delta} \frac{t / 2 \Delta}{\omega-\Delta+i \eta}\right]
\end{aligned}
$$

where $i+\delta$ refers to the $\mathrm{NN}$ sites of $i$. The correction to density results from the spectral weight transferred to the upper band,

$$
\delta n_{i \uparrow}^{(1)}=\int \frac{d \omega}{2 \pi i} e^{i \omega \eta}\left[\delta g_{\uparrow}^{(1)}\right]_{i i}=-2 \frac{t^{2}}{\Delta^{2}}\left(\frac{\epsilon_{i}-\sum_{\delta} \epsilon_{i+\delta} / 4}{U}\right) .
$$

Thus for positive $\epsilon_{i}$ (and $\epsilon_{i+\delta}=0$, for the sake of argument) the electron on site $i$ is more delocalized as its energy is pushed up, leading to the above decrease in density. A simple way to see this is in terms of the escape probability for the spin-up electron from site $i$ to its nearest neighbors $i+\delta$ due to the virtual hopping process. The net probability of escape changes from $t^{2} / U^{2}$ to $(1 / 4) \Sigma_{\delta} t^{2} /\left(U+\epsilon_{i+\delta}\right.$ $\left.-\epsilon_{i}\right)^{2}$, which to first order in $\epsilon$ precisely yields the above result.

From the particle-hole symmetry with spin flip in the Hubbard antiferromagnet, ${ }^{46}$ the spin-down hole experiences the same effect, except that the potentials are reversed for the hole. Therefore, there is an increase of identical magnitude in the spin-down hole density, implying an identical decrease in the spin-down particle density. This leads to a vanishing change in the local magnetization $m_{\mathrm{HF}}$, and the particle-hole propagator $\chi^{0}$. This cancellation would be absent if the random potential was spin dependent, and is connected to the time-reversal symmetry. Thus,

$$
\delta m_{\mathrm{HF}}^{(1)}=0 ; \quad\left[\delta \chi^{0}\right]^{(1)}=0 .
$$

\section{Second order}

Second-order changes in densities $\delta n_{i \sigma}^{(2)}$ are similarly obtained from the second-order correction $g_{\sigma}^{0} V g_{\sigma}^{0} V g_{\sigma}^{0}$. As this term is invariant under the transformation $V \rightarrow-V$, identical 
changes are obtained for spin-up electron and spin-down hole. The changes in spin-up and spin-down electronic densities are therefore equal and opposite, and hence the change in local magnetization adds up to

$$
\delta m_{i}^{(2)}=-6 \frac{t^{2}}{\Delta^{2}} \frac{1}{4} \sum_{\delta} \frac{\left(\epsilon_{i}-\epsilon_{i+\delta}\right)^{2}}{U^{2}} .
$$

This result also follows from the second-order correction to the net escape probability, discussed earlier below Eq. (A4). Configuration averaging, with $\left\langle\epsilon^{2}\right\rangle=W^{2} / 12$, therefore yields the following quadratic decrease of sublattice magnetization with disorder, which is suppressed by the small factor $t^{2} / U^{2}$. As discussed in Sec. III, quantum spin-fluctuation effects do not substantially modify this result, which qualitatively agrees with the quantum Monte Carlo studies of the disordered Hubbard model ${ }^{41}$

$$
\left\langle\delta m_{\mathrm{HF}}^{(2)}\right\rangle=-4 \frac{t^{2}}{U^{2}} \frac{W^{2}}{U^{2}} .
$$

*On leave from Department of Physics, Indian Institute of Technology, Kanpur 208016.

${ }^{1}$ D. Vaknin, S. K. Sinha, D. E. Moncton, D. C. Johnston, J. M. Newsam, C. R. Safinya, and H. E. King, Jr., Phys. Rev. Lett. 58, 2802 (1987).

${ }^{2}$ J. G. Bednorz and K. A. Müller, Z. Phys. B 64, 188 (1986).

${ }^{3}$ A. P. Kampf, Phys. Rep. 249, 219 (1994); W. Brenig, ibid. 251, 153 (1995)

${ }^{4}$ K. B. Lyons, P. A. Fleury, J. P. Remeika, A. S. Cooper, and T. J. Negran, Phys. Rev. B 37, 2353 (1988).

${ }^{5}$ R. R. P. Singh, P. A. Fleury, K. B. Lyons, and P. E. Sulewski, Phys. Rev. Lett. 62, 2736 (1989).

${ }^{6}$ S. Chakravarty, B. I. Halperin, and D. R. Nelson, Phys. Rev. Lett. 60, 1057 (1988): Phys. Rev. B 39, 2344 (1988).

${ }^{7}$ A. Singh, Curr. Sci. 62, 404 (1992).

${ }^{8}$ G. R. Stewart, Rev. Mod. Phys. 56, 755 (1984).

${ }^{9} \mathrm{P}$. W. Anderson, in Frontiers and Borderlines in Many-Particle Physics, edited by R. A. Broglia and J. R. Schrieffer (NorthHolland, Amsterdam, 1988), p. 1.

${ }^{10}$ Metal-Insulator Transitions Revisited, edited by P. P. Edwards and C. N. R. Rao (Taylor \& Francis, London, 1995)

${ }^{11}$ H. R. Ott, in Progress in Low Temperature Physics, edited by D. F. Brewer (North-Holland, Amsterdam, 1987), Vol. XI, p. 215.

${ }^{12}$ B. L. Altshuler and A. G. Aronov, Pis'ma Zh. Éksp. Teor. Fiz. 27, 700 (1978) [JETP Lett. 27, 662 (1978)]; for a review, see P. A. Lee and T. V. Ramakrishnan, Rev. Mod. Phys. 57, 287 (1985).

${ }^{13}$ K. P. Rajeev and A. K. Raychaoudhury, Phys. Rev. B 46, 1309 (1992); A. K. Raychaudhury, K. P. Rajeev, H. Srikanth, and R. Mahendiran, Physica B 197, 124 (1994).

${ }^{14}$ A. K. Raychaudhury, Phys. Rev. B 44, 8572 (1991).

${ }^{15}$ N. Bulut, D. Hone, D. J. Scalapino, and E. Y. Loh, Phys. Rev. Lett. 62, 2192 (1989).

${ }^{16}$ W. Brenig and A. P. Kampf, Phys. Rev. B 43, 12914 (1991).

${ }^{17}$ E. Manousakis, Phys. Rev. B 45, 7570 (1992).

${ }^{18}$ C. C. Wan, A. B. Harris, and D. Kumar, Phys. Rev. B 48, 1036 (1993).

${ }^{19}$ D. Poilblanc, D. J. Scalapino, and W. Hanke, Phys. Rev. Lett. 72, 884 (1994).
Turning now to the second-order corrections to $\chi^{0}$, we find that processes containing energy renormalization in the fermionic propagators (either solely, or along with density renormalization) cancel, so that the net result to order $(W / U)^{2}$ is

$$
\begin{gathered}
{\left[\delta \chi^{0}\right]_{i i}^{(2)}=-\frac{t^{2}}{\Delta^{3}} \frac{1}{4} \sum_{\delta} \frac{\left(\epsilon_{i}-\epsilon_{i+\delta}\right)^{2}}{U^{2}},} \\
{\left[\delta \chi^{0}\right]_{i, i+\delta}^{(2)}=-\frac{t^{2}}{\Delta^{3}} \frac{1}{4} \frac{\left(\epsilon_{i}-\epsilon_{i+\delta}\right)^{2}}{U^{2}} .}
\end{gathered}
$$

We note that the sum of all NN matrix elements is precisely the diagonal-matrix element. An immediate consequence of this correlation is that the Goldstone mode is preserved, as expected from spin-rotational symmetry, and that generally the effective scattering of low-energy, long-wavelength magnon modes is weak. This disorder-induced perturbation $\delta \chi^{0}$ directly yields the magnon self-energy, and disorder renormalization effects on magnon properties are discussed in Secs. III and IV.

${ }^{20}$ P. Sen, S. Basu, and A. Singh, Phys. Rev. B 50, 10381 (1994).

${ }^{21}$ P. Sen and A. Singh, Phys. Rev. B 53, 328 (1996).

${ }^{22}$ A. Singh and P. Sen, Phys. Rev. B 57, 10598 (1998).

${ }^{23}$ R. J. Birgeneau, Y. Endoh, Y. Hidaka, and K. Kakurai, in Mechanisms of High Temperature Superconductivity, edited by H. Kamimura and A. Oshiyama (Springer-Verlag, Heidelberg, 1989).

${ }^{24}$ C. Castellani, G. Kotliar, and P. A. Lee, Phys. Rev. Lett. 59, 323 (1987).

${ }^{25}$ D. Belitz and T. R. Kirkpatrick, Rev. Mod. Phys. 66, 261 (1994).

${ }^{26}$ M. Milovanović, S. Sachdev, and R. N. Bhatt, Phys. Rev. Lett. 63, 82 (1989).

${ }^{27}$ A. Langenfeld and P. Wölfle, Ann. Phys. (Leipzig) 4, 43 (1995).

${ }^{28}$ S. Sachdev, Philos. Trans. R. Soc. London, Ser. A 356, 173 (1998).

${ }^{29}$ W. L. McMillan, Phys. Rev. B 24, 2739 (1981).

${ }^{30}$ M. Ma and E. Fradkin, Phys. Rev. B 28, 2990 (1983).

${ }^{31}$ A. M. Finkelshtein, Zh. Éksp. Teor. Fiz. 84, 168 (1983) [Sov. Phys. JETP 57, 97 (1983)].

${ }^{32}$ C. Castellani, C. Di Castro, P. A. Lee, and M. Ma, Phys. Rev. B 30, 527 (1984); C. Castellani, C. Di Castro, and M. Grilli, ibid. 34, 5907 (1986).

${ }^{33}$ A. M. Finkelshtein, Z. Phys. B 56, 189 (1984)

${ }^{34}$ C. Castellani, C. Di Castro, P. A. Lee, M. Ma, S. Sorella, and E. Tabet, Phys. Rev. B 30, 1596 (1984); 33, 6169 (1986).

${ }^{35}$ M. Ma, Phys. Rev. B 26, 5097 (1982).

${ }^{36}$ J. Yi, L. Zhang, and G. S. Canright, Phys. Rev. B 49, 15920 (1994).

${ }^{37}$ G. T. Zimanyi and E. Abrahams, Phys. Rev. Lett. 64, 2719 (1990).

${ }^{38}$ V. Janiš and D. Vollhardt, Phys. Rev. B 46, 15712 (1992).

${ }^{39}$ V. Dobrosavljević and G. Kotliar, Phys. Rev. Lett. 71, 3218 (1993); Phys. Rev. B 50, 1430 (1994).

${ }^{40}$ V. Janiš, M. Ulmke, and D. Vollhardt, Europhys. Lett. 24, 287 (1993); M. Ulmke, V. Janiś, and D. Vollhardt, Phys. Rev. B 51, 10411 (1995).

${ }^{41}$ M. Ulmke and R. T. Scalettar, Phys. Rev. B 55, 4149 (1997).

${ }^{42}$ M. A. Tusch and D. E. Logan, Phys. Rev. B 48, 14843 (1993); 
51, 11940 (1995); D. G. Rowan, Y. H. Szczech, M. A. Tusch, and D. E. Logan, J. Phys.: Condens. Matter 7, 6853 (1995).

${ }^{43}$ Y. H. Szczech, M. A. Tusch, and D. E. Logan, J. Phys.: Condens. Matter 9, 9621 (1997).

${ }^{44}$ Y. H. Szczech, M. A. Tusch, and D. E. Logan, J. Phys.: Condens. Matter 10, 639 (1998).

${ }^{45}$ A. Singh, Phys. Rev. B 43, 3617 (1991).

${ }^{46}$ A. Singh and Z. Tešanović, Phys. Rev. B 41, 614 (1990); 41, 11 457 (1990).

${ }^{47}$ S. Basu and A. Singh, Phys. Rev. B 53, 6406 (1996).
${ }^{48}$ P. W. Anderson, Phys. Rev. 86, 694 (1952).

${ }^{49}$ T. Oguchi, Phys. Rev. 117, 117 (1960).

${ }^{50}$ The coefficient of $x$ was found to be nearly 3 for long-wavelength magnon modes within a $T$-matrix analysis of spin vacancies in the QHAF (Ref. 16).

${ }^{51}$ A. Singh, cond-mat/9802049 (unpublished).

${ }^{52}$ W. Hofstetter and D. Vollhardt, Ann. Phys. (Leipzig) 7, 48 (1998).

${ }^{53}$ M. Jarrell and J. E. Gubernatis, Phys. Rep. 269, 133 (1996).

${ }^{54}$ A. Singh, M. Ulmke, and D. Vollhardt (unpublished). 